\title{
CagA increases DNA methylation and decreases PTEN expression in human gastric cancer
}

\author{
BAOGUI ZHANG ${ }^{1 *}$, XIAOBEI ZHANG ${ }^{2 *}$, MENG JIN $^{1 *}$, LEI HU $^{3}$, MINGDE ZANG $^{4,5}$, \\ WEILONG QIU ${ }^{1}$, SHOUQI WANG ${ }^{1}$, BINGYA LIU ${ }^{6}$, SHIQI LIU ${ }^{1}$ and DONGLI GUO ${ }^{1}$
}

\begin{abstract}
Departments of ${ }^{1}$ Gastrointestinal Surgery and ${ }^{2}$ Central Laboratory, Affiliated Hospital of Jining Medical University, Jining, Shandong 272000; ${ }^{3}$ Department of General Surgery Affiliated Provincial Hospital of Anhui Medical University, Hefei, Anhui 230001; ${ }^{4}$ Department of Gastric Cancer Surgery, Fudan University Shanghai Cancer Center; ${ }^{5}$ Department of Oncology, Shanghai Medical College, Fudan University, Shanghai 200032; ${ }^{6}$ Department of Surgery, Shanghai Key Laboratory of Gastric Neoplasms, Shanghai Institute of Digestive Surgery, Ruijin Hospital, Shanghai Jiao Tong University School of Medicine, Shanghai 200025, P.R. China
\end{abstract}

Received April 28, 2018; Accepted September 28, 2018

DOI: $10.3892 / \mathrm{mmr} .2018 .9654$

\begin{abstract}
Gastric cancer is one of the leading causes of cancer-associated mortality worldwide. Cytotoxin-associated gene A (CagA) has been reported to be associated with gastric diseases. Phosphatase and tensin homolog (PTEN) and tet methylcytosine dioxygenase 1 (Tet1) are important tumor-suppressor genes. The present study aimed to investigate the underlying functions of $\mathrm{Cag} \mathrm{A}$ in human gastric cancer, and to explore the associations between CagA, PTEN and Tet1 in gastric cancer. For that purpose, CagA overexpression and Tet1 interference recombinant lentiviral plasmids were constructed. Quantitative polymerase chain reaction (qPCR) was utilized to screen gene expression in HGC-27 human gastric cancer cells overexpressing CagA. qPCR and western blotting were used to detect gene and protein expression, respectively. In addition, the methylation status of PTEN was detected by methylation-specific PCR. The expression levels of PTEN, Tet1, apolipoprotein B mRNA editing enzyme catalytic subunit (APOBEC)3A, APOBEC3C and APOBEC3F were significantly decreased in the CagA overexpression group compared with in the negative control group in HGC-27 cells. Compared with in the negative control group, the mRNA and protein expression levels of PTEN were markedly decreased in cells with Tet1 interference. The decreased expression of PTEN was associated with increased methylation levels in the
\end{abstract}

Correspondence to: Dr Shiqi Liu or Dr Dongli Guo, Department of Gastrointestinal Surgery, Affiliated Hospital of Jining Medical University, 89 Guhuai Street, Jining, Shandong 272000, P.R. China E-mail: drliushiqi@163.com

E-mail: gd119630809@163.com

*Contributed equally

Key words: CagA, gastric cancer, Tet1, PTEN, methylation cells. In addition, the protein expression levels of PTEN were significantly decreased in HGC-27 cells when CagA was overexpressed. The expression levels of PTEN and Tet1 were also markedly decreased in $\mathrm{CagA}^{+}$gastric cancer tissues compared with in non-cancerous tissues. The decreased expression of PTEN in $\mathrm{CagA}^{+}$gastric cancer tissues was associated with increased methylation levels. In conclusion, overexpression of CagA significantly decreased the expression of PTEN, Tet1, APOBEC3A, APOBEC3C and APOBEC3F in human gastric cancer. In addition, CagA increased DNA methylation and decreased PTEN expression, which was reversed by Tetl overexpression. The present study may facilitate future therapeutic approaches targeting human gastric cancer.

\section{Introduction}

Gastric cancer is one of the most common types of cancer worldwide. The global incidence of gastric cancer has declined over recent decades (1); however, due to aging in the world population, the absolute number of new cases per year is increasing. Furthermore, there is a recent upward trend in young patients with gastric cancer (2); therefore, gastric cancer remains an important cause of cancer-associated mortality.

Cytotoxin-associated gene A (CagA) has been reported to be associated with gastric diseases, particularly peptic ulcer disease (3). In total, 85-100\% patients with duodenal ulcers are infected with $\mathrm{CagA}^{+}$Helicobacter pylori (H. pylori) strains (4). The origin of $H$. pylori strains has been reported to serve an important role in the rates of gastric cancer, which may be associated with differences in the capacity of different strains to express CagA. Notably, the incidence of precancerous lesions and gastric cancer is higher in patients with $\mathrm{CagA}^{+}$strains (5). Furthermore, $88 \%$ of animals infected with a rodent-adapted strain of CagA-producing human $H$. pylori developed gastric dysplasia within 4 weeks, and $75 \%$ of animals developed gastric adenocarcinoma by week 8 (6). In addition, specific amino acid sequences in the CagA protein have been revealed to be associated with an increased risk of malignancy (7); 
however, the mechanism by which CagA affects the expression of genes in gastric cancer remains elusive.

The phosphatase and tensin homolog (PTEN) gene, which is located on chromosome $10 \mathrm{q} 23$, is a tumor-suppressor gene (8-10). It has been reported to regulate the phosphoinositide-3-kinase-protein kinase B (AKT) and mechanistic target of rapamycin signaling pathways, which serve important roles in apoptosis, cell cycle progression and cell proliferation. Loss of function of PTEN has been reported to result in oncogenesis and somatic mutations in various malignancies (11). Tet methylcytosine dioxygenase (Tet)1 has been reported to exert its tumor-suppressor function in gastric cancer through interactions with the $\mathrm{p} 53$-enhancer of zeste 2 polycomb repressive complex 2 subunit (EZH2) signaling pathway. Tet1 suppresses cancer formation via inhibition of the oncogenic protein EZH2 and activation of p53, possibly via DNA demethylation (12). However, the associations between CagA, PTEN and Tet1 in gastric cancer remain unclear. Therefore, the present study aimed to investigate the interactions between CagA, PTEN and Tet1 in gastric cancer.

\section{Materials and methods}

Reagents. Primers, TRIzol ${ }^{\circledR}$ reagent, SuperScript III Reverse Transcriptase, SYBR Green I and DEPC $\mathrm{H}_{2} \mathrm{O}$ were purchased from Invitrogen (Thermo Fisher Scientific, Inc., Waltham, MA, USA). The RNase inhibitor was purchased from Fermentas (Thermo Fisher Scientific, Inc., Pittsburgh, PA, USA). Platinum Taq DNA polymerase, oligo dT/primer and $100 \mathrm{mM}$ dNTPs were purchased from Invitrogen (Thermo Fisher Scientific, Inc.) and used for PCR. Dulbecco modified Eagle's medium (DMEM) was purchased from Gibco (Thermo Fisher Scientific, Inc.).

Construction of recombinant plasmids and lentiviral packaging. HEK 293T cells (American Type Culture Collection, Manassas, VA, USA) and human gastric cancer cell line HGC-27 cells were cultivated in Dulbecco modified Eagle's medium (DMEM, Invitrogen; Thermo Fisher Scientific, Inc.) with $10 \%$ of fetal bovine serum (FBS; Invitrogen; Thermo Fisher Scientific, Inc.) in a humidified incubator with $5 \% \mathrm{CO}_{2}$ at $37^{\circ} \mathrm{C}$. The human gastric cancer cell line HGC-27 was purchased from RIKEN Bioresource Center (Tsukuba, Japan); this cell line is derived from a metastatic lymph node, and is a highly metastatic cell line due to its undifferentiated characteristics. The Tet1 catalytic domain (NM_030625.2) was used to design three short hairpin RNAs (shRNAs) (Table I). Upon annealing, shRNA was treated with T4 DNA polymerase in the presence of dATP, and then annealed with the pds019-p16.3-SHRNA-BSD vector (Novagen, EMD Millipore, Billerica, MA, USA). Then, the annealed complex was transformed into $E$. coli host strain. The recombinant plasmids were sequenced, and named CL946-1, CL946-2 and CL946-3. In addition, the Tet1 catalytic domain was cloned into a PDS087_pL6-TO-V5-GIM vector (Novagen; EMD Millipore) using the restriction enzyme site $N h e \mathrm{I} / A s c \mathrm{I}$. The recombinant plasmid was named CL981_pL6-TO-V5-tet1-CD. Similarly, a CagA (AB015416.1) overexpression vector was generated. Subsequently, packaging mix (9 $\mu \mathrm{g}$; Thermo Fisher Scientific, Inc.) and recombinant lentiviral plasmids (3 $\mu \mathrm{g})$ were added
Table I. Sequences of shRNAs used for vector construction and primers used for reverse transcription-quantitative polymerase chain reaction.

Primer/shRNA Sequence (5'-3')

Sh1-Tet1-F

CACCGCAGCTAATGAAGGTCC

CCAGAACGAATTCTGGACCTT

CATTAGCTGC

Sh1-Tet1-R

AAAAGCAGCTAATGAAGGTC CAGAATTCGTTCTGGACTAGC TGCCTTCAT

Sh2-Tet1-F CACCG*CCCAGAAGATTTAGA ATTGATCGAAATCAATTCTAA ATCTTCTGGG

Sh2-Tet1-R AAAACCCAGAAGATTTAGAAT TGATTTCGATCAATTCTAAATC TTCTGGGC*

Sh3-Tet1-F CACCG*CCTCCAGTCTTAATAA GGTTACGAATAACCTTATTAAG ACTGGAGG

Sh3-Tet1-R

AAAACCTCCAGTCTTAATAAG GTTATTCGTAACCTTATTAAGA CTGGAGGC

Tet1-F

Tet1-R

Negative control-F

AGTGGTGACTATGCCAGTGC CAGACCCCACATCGCTTTCT

Negative control-R

TAATTCTCCGAACGTGTCACG TTTCAAGAGAACGTGACACG TTCGGAGAATTTTTTTT

TCGAGAAAAAAAATTCTCCGA AGCGTGTCACGTTCTCTTGAA ACTGACACGTTCGGAGTTA

PTEN-F PTEN-R

CagA-F

CagA-R

Ras-F

Ras-R

c-met-F

c-met-R

c-myc-F

c-myc-R

ESR2-F

ESR2-R

Tet2-F

Tet2-R

Tet3-F

Tet3-R

ACRC-F

ACRC-R

AICDA-F

AICDA-R

ALKBH1-F

ALKBH1-R

ALKBH2-F

ALKBH2-R
CTCAGCCGTTACCTGTGTGT

AGGTTTCCTCTGGTCCTGGT

CTGCCAAACAATCTTTTGCAG

CTGGTTCTTGATTGATTGCTTC

ACTGGGGAGGGCTTTCTTTG

GCATCATCAACACCCTGTCT

GTCCTGCAGTCAATGCCTCT

GTCAGCCTTGTCCCTCCTTC

CATCAGCACAACTACGCAGC

GCTGGTGCATTTTCGGTTGT

GTCCCTGGTGTGAAGCAAGA

CCTTCACACGACCAGACTCC

TAGCCACACCCCAGCTTTAG

TCTGGATAAACGCCATGTGTCT

GTGCTCCGGAAGAGTTTCCA

ACGGTGCACCCATTGTAGAG

CAGCACTGGTGAGATGTGGT

CATCAATCAGCCAGGAGGCA

AGCCTCTTGATGAACCGGAG

TGTCACGCCTCTTCACTACG

ACTGGGTGAAACAGTGCCTT

AAACTTCGGGGTCTCCGTTT

GCTGGGCTGACCTACACATT

CGATGTGGTCACAGCCATCT 
Table I. Continued.

\begin{tabular}{ll}
\hline Primer/shRNA & \multicolumn{1}{c}{ Sequence (5'-3') } \\
\hline APEX1-F & TTACGGCATAGGCGATGAGG \\
APEX1-R & TGTGCCACATTGAGGTCTCC \\
APOBEC2-F & CGTGTGCTGACCGCATTATC \\
APOBEC2-R & CAGTTTACAGCCAGCCTCCT \\
APOBEC3A-F & GGTCAAGATGGACCAGCACA \\
APOBEC3A-R & AGGAACCAGGTCCAAGAAGC \\
APOBEC3C-F & ACCTATGGGAAGCCAACGAT \\
APOBEC3C-R & AGAGAGGAAGCACCTTTCTGC \\
APOBEC3F-F & GTCTGGCTGTGCTACGAAGT \\
APOBEC3F-R & CACATTTCTGCGTGGTGCTC \\
FTO-F & ACCCATGGCTCAACTGGAAG \\
FTO-R & AGCAGGTAATGTTCGGGCAA \\
TDG-F & GAGAACGCGGGCAGCTATTC \\
TDG-R & TGGGGCTGGAACTTCTTCTG
\end{tabular}

ACRC, acidic repeat containing; AICDA, activation-induced cytidine deaminase; ALKBH1, alkB homolog 1, histone H2A dioxygenase; ALKBH2, alkB homolog 2, $\alpha$-ketoglutarate dependent dioxygenase; APOBEC, apolipoprotein B mRNA editing enzyme catalytic subunit; CagA, cytotoxin-associated gene A; ESR2, estrogen receptor 2; F, forward; FTO, FTO, $\alpha$-ketoglutarate dependent dioxygenase; PTEN, phosphatase and tensin homolog; R, reverse; sh/shRNA, short hairpin RNA; Tet, tet methylcytosine dioxygenase; TDG, thymine DNA glycosylase.

into Opti-Minimum Essential Medium (MEM, Thermo Fisher Scientific, Inc.) and mixed. In addition, Lipofectamine ${ }^{\circledR} 2000$ (36 $\mu$; Invitrogen; Thermo Fisher Scientific, Inc.) was mixed with Opti-MEM $(1.5 \mathrm{ml})$, and incubated at room temperature for $5 \mathrm{~min}$. The plasmid solution and diluted Lipofectamine ${ }^{\circledR}$ 2000 were then mixed, and incubated at room temperature for $5 \mathrm{~min}$. The mixture was added into a culture dish with HEK 293T cells, and the cells were cultured for $48 \mathrm{~h}$ in the conditions described above. The cell supernatant was then collected, centrifuged at $1,500 \mathrm{x}$ g for $10 \mathrm{~min}$ at room temperature, and filtered. The virus solution was then condensed by centrifuging at $50,000 \times \mathrm{g}$ for $2 \mathrm{~h}$ at $4^{\circ} \mathrm{C}$, and resuspended in DMEM. Tet1 interference (VL946_PDS19-shTET1), Tet1 interference (VL946_PDS19-shTET2), Tet1 interference (VL946_PDS19-shTET3), Tet1 overexpression (LV576_ pL6-TO-V5-tet1-CD), CagA overexpression and negative control viruses were derived. HGC-27 cells were maintained in DMEM with $10 \%$ of fetal bovine serum in a humidified incubator with $5 \% \mathrm{CO}_{2}$ at $37^{\circ} \mathrm{C}$ and were plated into cell plates $(60 \mathrm{~mm})$ at $4 \times 10^{5}$ cells/well overnight. HGC-27 cells were infected with CL946-1, CL946-2 and CL946-3 viruses for 3 days and quantitative polymerase chain reaction (qPCR) was used to detect interference efficiency. To determine the viruses transduction efficiency in HGC-27 cells, GFP expression was examined by microscopy at different multiplicities of infection (MOIs) on day 3 following infection.

Cell viability assay. Cell viability was measured using the Cell Counting kit 8 (CCK-8; Dojindo Molecular Technologies, Inc.,

Table II. Primers used for methylation-specific polymerase chain reaction.

Primer Sequences (5'-3')

PTEN-M-F

PTEN-M-R

PTEN-U-F

PTEN-U-R

GTATTTCGAGTAAAGGAAGAAGACG
GATAAAAAACTACAACCCAACGAA
TATTTTGAGTAAAGGAAGAAGATGA
CAATAAAAAACTACAACCCAACAAA

F, forward; M, methylated; PTEN, phosphatase and tensin homolog; $\mathrm{R}$, reverse; $\mathrm{U}$, unmethylated.

Table III. Clinical information of patients with gastric cancer.

\begin{tabular}{llccc}
\hline Sample no. & Sex & Age (years) & TNM stage & CagA \\
\hline 1 & Male & 45 & T3N1M0 & + \\
2 & Male & 71 & T2N0M0 & - \\
3 & Male & 52 & T4N3M0 & + \\
4 & Female & 55 & T4N3M0 & + \\
5 & Male & 64 & T3N1M0 & - \\
6 & Male & 59 & T2N0M0 & - \\
7 & Male & 71 & T3N2M0 & + \\
8 & Male & 53 & T4N3M0 & + \\
9 & Male & 74 & T4N3M0 & + \\
10 & Female & 40 & T2N0M0 & - \\
11 & Male & 72 & T3N2M0 & + \\
12 & Male & 74 & T2N0M0 & - \\
\hline
\end{tabular}

CagA, cytotoxin-associated gene A.

Kumamoto, Japan). Briefly, HGC-27 cells ( $5 \times 10^{3}$ cells/well) were seeded into 96 -well plates and cultured overnight. Subsequently, cells were infected with TET1 interference or CagA overexpression viruses for 24,48 or $72 \mathrm{~h}$. The medium was then removed and $10 \mu \mathrm{l} \mathrm{CCK}-8$ solution was added to each well. After $4 \mathrm{~h}$, cell viability was detected using a plate reader at a wavelength of $450 \mathrm{~nm}$.

Reverse transcription (RT)-qPCR. The mRNA expression levels of PTEN, Tet1, apolipoprotein B mRNA editing enzyme catalytic subunit (APOBEC)3A, APOBEC3C, APOBEC3F, Ras, c-myc, c-met, estrogen receptor 2 (ESR2), Tet2, Tet3, acidic repeat containing (ACRC), activation-induced cytidine deaminase (AICDA), alkB homolog 1, histone H2A dioxygenase (ALKBH1), alkB homolog 2, $\alpha$-ketoglutarate dependent dioxygenase (ALKBH2), apurinic/apyrimidinic endodeoxyribonuclease 1 (APEX1), APOBEC2, FTO, $\alpha$-ketoglutarate dependent dioxygenase (FTO) and thymine DNA glycosylase (TDG) in HGC-27 human gastric carcinoma cells were detected by RT-qPCR. All these genes have been reported to have close associations with gene methylation/demethylation (13). Total RNA was extracted using TRIzol ${ }^{\circledR}$ reagent, according to the manufacturer's protocol. A universal cDNA synthesis kit (Invitrogen; Thermo Fisher Scientific, Inc.) was used for RT. 

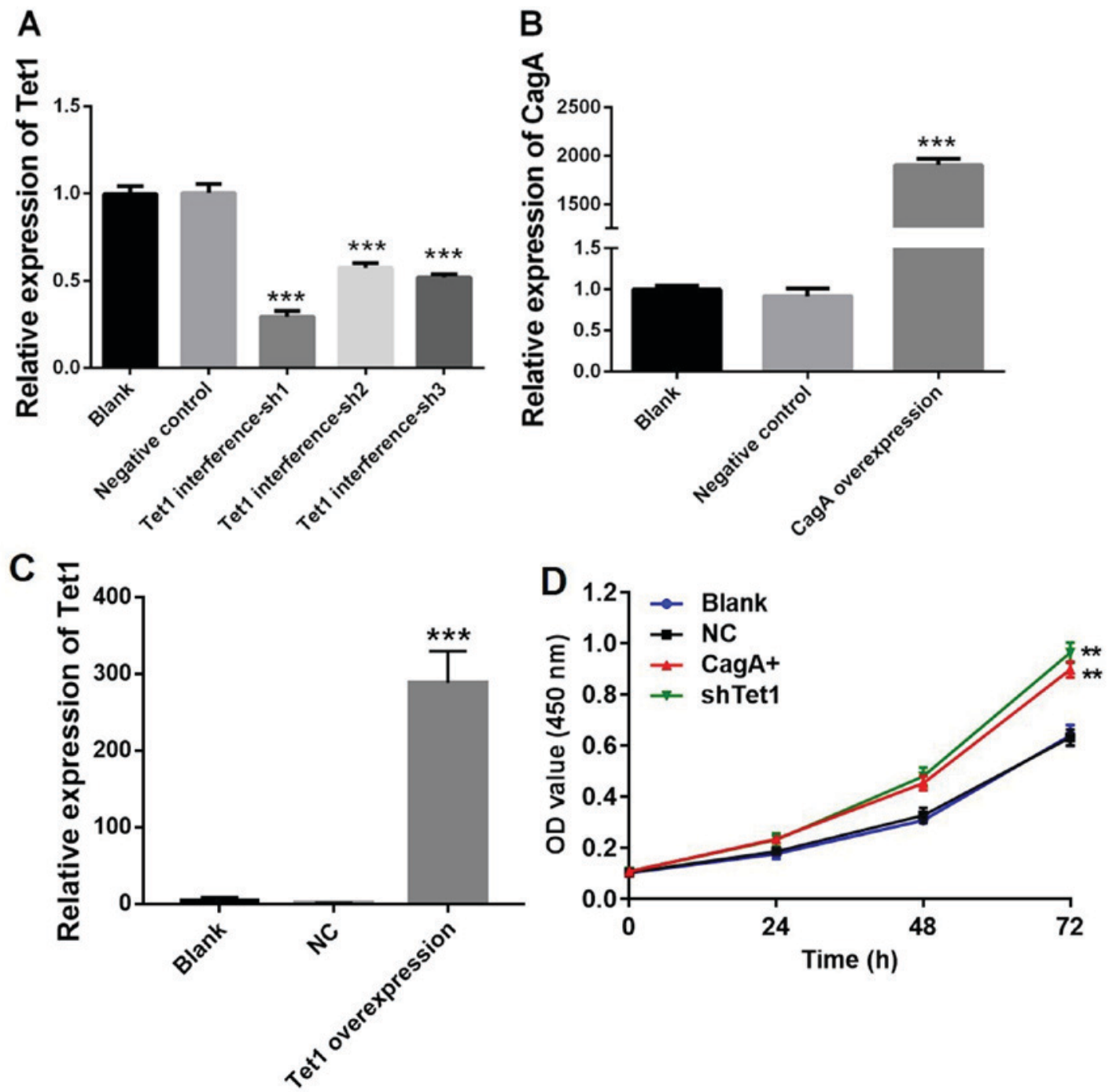

Figure 1. Confirmation of Tet1 interference, Tet1 overexpression and CagA overexpression in HGC-27 cells. (A) HGC-27 cells were infected with viruses carrying Tet1 interference vectors. Relative expression was detected by RT-qPCR. Expression levels of Tet1 were significantly decreased in HGC-27 cells upon Tet1 interference. (B and C) HGC-27 cells were infected with viruses carrying a CagA or Tet1 overexpression vector. Relative expression levels of CagA and Tet1 were detected by RT-qPCR. Compared with in the NC group, CagA and Tet1 expression was markedly increased in the CagA and Tet1 overexpression groups, respectively. (D) HGC-27 cells were infected with viruses carrying Tet1 interference or CagA overexpression vectors for 24,48 or 72 h. Cell viability was detected using the Cell Counting kit 8 kit. These results indicated that Tet1 interference, Tet1 overexpression and CagA overexpression were successfully established. ${ }^{* * * *} \mathrm{P}<0.001,{ }^{* *} \mathrm{P}<0.01$ vs. the NC group $(\mathrm{n}=3)$. CagA, cytotoxin-associated gene A; NC, negative control; OD, optical density; RT-qPCR, reverse transcription-quantitative polymerase chain reaction; sh, short hairpin RNA; Tet1, tet methylcytosine dioxygenase 1.

Each reaction contained $0.5 \mu \mathrm{l}$ random primers $(0.2 \mu \mathrm{g} / \mu \mathrm{l})$ and $1 \mu \mathrm{l}$ SuperScript III reverse transcriptase $(200 \mathrm{U} / \mu \mathrm{l})$. The specific primers used for RT-qPCR are listed in Table I. PCR was performed using a SYBR qPCR kit (Invitrogen; Thermo Fisher Scientific, Inc.). The PCR conditions were as follows: Predenaturation at $95^{\circ} \mathrm{C}$ for $2 \mathrm{~min}$, followed by 40 cycles of denaturation at $95^{\circ} \mathrm{C}$ for $10 \mathrm{sec}$, annealing at $60^{\circ} \mathrm{C}$ for $30 \mathrm{sec}$ and polymerization at $70^{\circ} \mathrm{C}$ for $45 \mathrm{sec}$, followed by 1 cycle of melting curve at $95^{\circ} \mathrm{C}$ for $15 \mathrm{sec}, 60^{\circ} \mathrm{C}$ for $60 \mathrm{sec}$ and $95^{\circ} \mathrm{C}$ for 15 sec. PCR was performed using a CFX96 Touch $^{\text {TM }}$ Real-time PCR Detection system (Bio-Rad Laboratories, Inc., Hercules, CA, USA). Gene expression was determined and normalized to $\beta$-actin. The primers for $\beta$-actin were as follows: Forward, 5'-AGGGAAATCGTGCGTGAC-3' and reverse, 5'-CGCTCA TTGCCGATAGTG-3'. The $2^{-\Delta \Delta C q}$ method was used to analyze PCR results (14). The fluorophore used for qPCR was included in the SYBR qPCR kit.
Detection of methylation status of PTEN by methylationspecific PCR. Promoter methylation of the PTEN gene was determined by methylation-specific PCR. Genomic DNA from HGC-27 cells as well as control specimens were subjected to bisulfite modifications using an EZ DNA Methylation-Gold kit (Zymo Research Corp., Irvine, CA, USA) in accordance with the manufacturer's protocol. DNA (500 ng) was treated with sodium hydrogen sulfite at $50^{\circ} \mathrm{C}$ for $16 \mathrm{~h}$. Unmethylated cytidine was deaminated and converted into uracil, whereas methylated cytidine remained unchanged. Based on these alterations, primers to amplify methylated and unmethylated PTEN sequences by PCR were designed using MethPrimer (http://www.urogene.org/cgi-bin/methprimer/methprimer.cgi; Table II). PCR was performed using the following conditions: An initial melting step of $10 \mathrm{~min}$ at $95^{\circ} \mathrm{C}$; followed by 50 cycles of $20 \mathrm{sec}$ at $95^{\circ} \mathrm{C}, 20 \mathrm{sec}$ at $59^{\circ} \mathrm{C}$ and $45 \mathrm{sec}$ at $72^{\circ} \mathrm{C}$ with a final elongation step of 4 min at $72^{\circ} \mathrm{C}$ in a CFX 96 Touch $^{\text {TM }}$ Real-time 

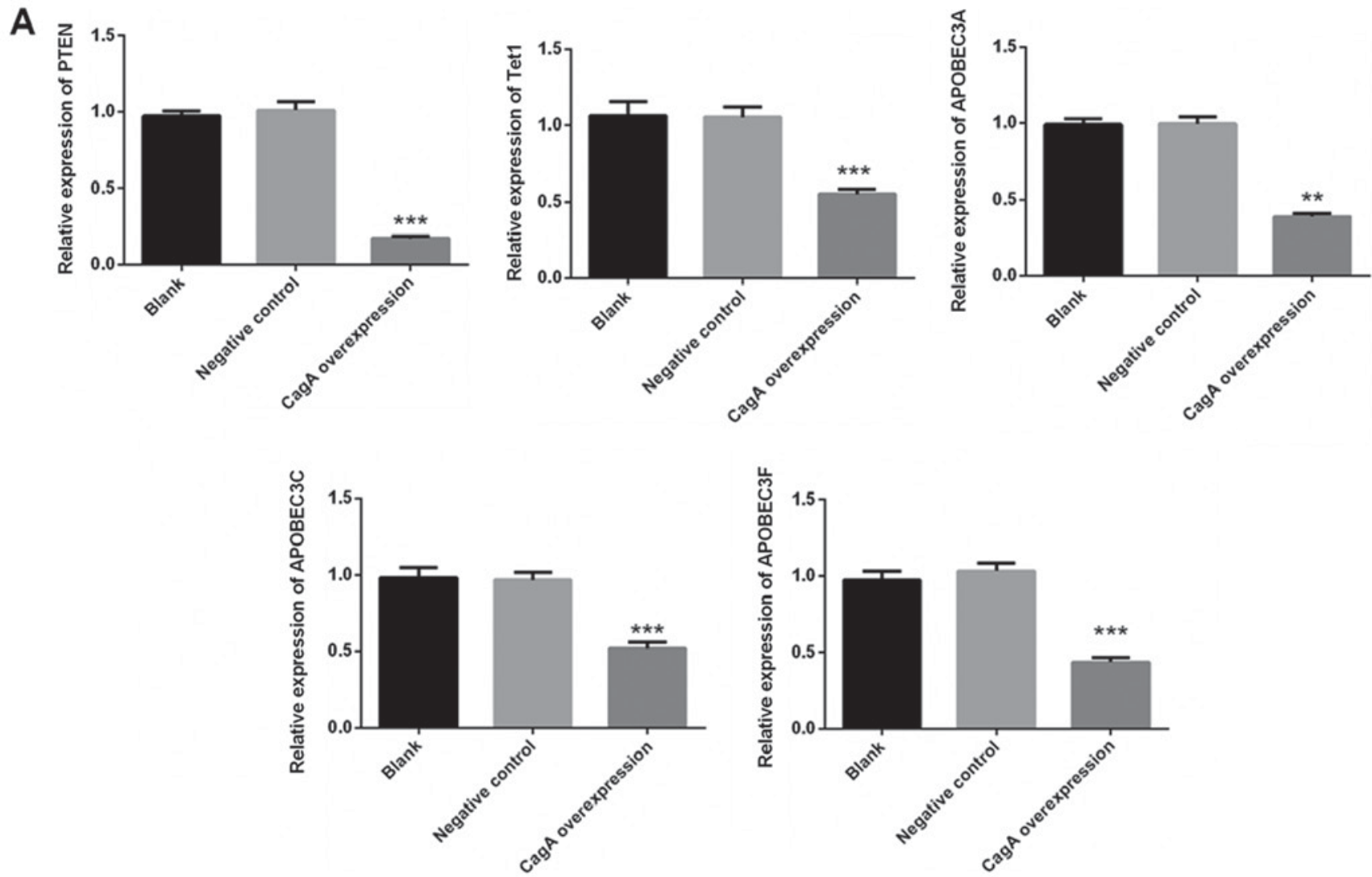

Figure 2. mRNA expression levels of PTEN, Tet1, APOBEC3A, APOBEC3C and APOBEC3F are significantly decreased in the CagA overexpression group. (A) HGC-27 cells were infected with viruses carrying a CagA overexpression vector. The expression levels of PTEN, Tet1, APOBEC3A, APOBEC3C and APOBEC $3 F$ were detected by reverse transcription-quantitative polymerase chain reaction. ${ }^{* *} \mathrm{P}<0.01,{ }^{* * *} \mathrm{P}<0.001$ vs. with the negative control group ( $\mathrm{n}=3$ ). APOBEC, apolipoprotein B mRNA editing enzyme catalytic subunit; CagA, cytotoxin-associated gene A; PTEN, phosphatase and tensin homolog; Tet1, tet methylcytosine dioxygenase 1 .

PCR Detection system (Bio-Rad Laboratories, Inc., Hercules, CA, USA). 3.5\% Agarose gel electrophoresis (Thermo Fisher Scientific, Inc.) was used to detect methylation status.

Western blot analysis. The protein expression levels of PTEN and Tet 1 were detected by western blot analysis. Cells or tissues were lysed in lysis buffer (cat. no. P0013; Beyotime Institute of Biotechnology, Haimen, China) with protease and phosphatase inhibitors (cat. no. P1045; Beyotime Institute of Biotechnology) at $4^{\circ} \mathrm{C}$. The lysis mixture was centrifuged at $10,000 \mathrm{x} \mathrm{g}$ for $10 \mathrm{~min}$ at $4^{\circ} \mathrm{C}$, and the supernatant containing cellular proteins was used in subsequent experiments. The protein concentration was determined using a bicinchoninic acid kit. Subsequently, proteins (40 $\mu \mathrm{g} / \mathrm{lane})$ were separated by $10 \%$ SDS-PAGE $(120 \mathrm{~V})$ and the separated proteins were transferred to polyvinylidene fluoride membranes (cat. no. FFP24; Beyotime Institute of Biotechnology) at $100 \mathrm{~V}$ for $2 \mathrm{~h}$. After blocking with 5\% non-fat milk for $1 \mathrm{~h}$ at room temperature, the membranes were incubated with primary antibodies against PTEN (1:400, cat. no. ab32199), Tet1 (1:400, cat. no. ab191698) and GAPDH (1:400, cat. no. ab8245) at $4{ }^{\circ} \mathrm{C}$ overnight. The membranes were then washed with Tris-buffered saline containing $0.5 \%$ Tween 20 , and were incubated with horseradish peroxidase-conjugated goat anti-rabbit secondary antibody $(1: 5,000$, cat. no. ab150077) at room temperature for $1 \mathrm{~h}$. All antibodies were purchased from Abcam (Cambridge, MA, USA). The membranes were then washed and incubated in enhanced chemiluminescence solution (cat. no. P0018A; Beyotime Institute of Biotechnology), and the images were captured on film (FF057; Beyotime Institute of Biotechnology) in a dark room. The experiments were repeated three times. Blot images were semi-quantified in grayscale using ImageJ (version 2.0; National Institutes of Health, Bethesda, MD, USA).

Gastric cancer tissue sample collection. A total of 12 patients with gastric cancer were enrolled between April 2017 and May 2018 for the study at the Affiliated Hospital of Jining Medical University (Jining, China), and gastric cancer was confirmed pathologically. Patient tumor tissues were obtained during surgery. All patients provided written informed consent and the study was approved by the Institutional Review Board/Ethics Committee of the Affiliated Hospital of Jining Medical University. The clinical information of the patients is provided in Table III.

Statistical analysis. Statistical data were analyzed by GraphPad Prism version 5.0 software (GraphPad Software, Inc., La Jolla, CA, USA). Data are presented as the means \pm standard deviation. Differences between more than two groups were compared by one-way analysis of variance followed by the Bonferroni post-hoc test. Differences between two groups were compared by Student's t-test. $\mathrm{P}<0.05$ was considered to indicate a statistically significant difference. 

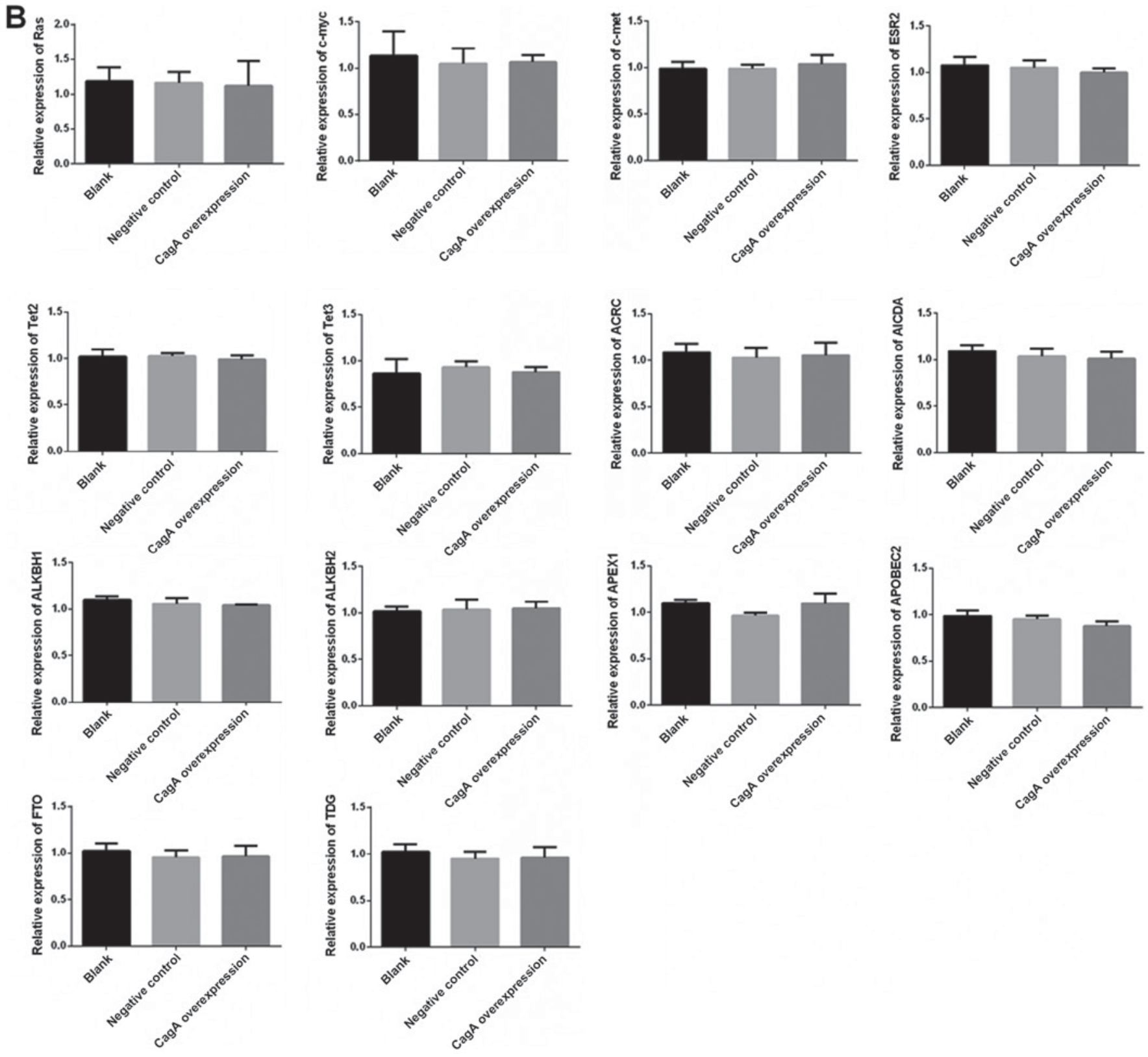

Figure 2. Continued. mRNA expression levels of PTEN, Tet1, APOBEC3A, APOBEC3C and APOBEC3F are significantly decreased in the CagA overexpression group. (B) Ras, c-myc, c-met, ESR2, Tet2, Tet3, ACRC, AICDA, ALKBH1, ALKBH2, APEX1, APOBEC2, FTO and TDG levels were detected following CagA overexpression in the cells. ACRC, acidic repeat containing; AICDA, activation-induced cytidine deaminase; ALKBH1, alkB homolog 1, histone $\mathrm{H} 2 \mathrm{~A}$ dioxygenase; ALKBH2, alkB homolog 2, $\alpha$-ketoglutarate dependent dioxygenase; APOBEC, apolipoprotein B mRNA editing enzyme catalytic subunit; CagA, cytotoxin-associated gene A; ESR2, estrogen receptor 2; FTO, FTO, $\alpha$-ketoglutarate dependent dioxygenase; PTEN, phosphatase and tensin homolog; Tet1, tet methylcytosine dioxygenase 1; TDG, thymine DNA glycosylase.

\section{Results}

Tet1 interference and CagA overexpression in HGC-27 cells. Initially, HGC-27 cells were infected with viruses carrying Tet1 interference or CagA overexpression vectors. Relative expression levels of Tet1 and CagA were detected by RT-qPCR. The expression levels of Tet1 in HGC-27 cells were significantly decreased by Tet1 interference compared with in the negative control group $(\mathrm{P}<0.001$; Fig. 1A). Since Tet1 interference-sh1 exerted the best inhibitory effect on the cells, it was used in subsequent experiments. In addition, compared with in the negative control group, infection of the cells with CagA or Tetl overexpression vectors markedly increased the expression of CagA and Tet1, respectively $(\mathrm{P}<0.001$; Fig. 1B and C). Furthermore, Tet1 interference or CagA overexpression significantly enhanced HGC-27 cell viability compared with in the control group $(\mathrm{P}<0.01$; Fig. 1D). These results indicated that Tet1 interference, Tet1 overexpression and CagA overexpression were successfully achieved.

Expression levels of PTEN, Tet1, APOBEC 3A, APOBEC $3 C$ and $A P O B E C 3 F$ are significantly decreased in the CagA overexpression group. RT-qPCR was used to detect gene expression in HGC-27 cells under the condition of CagA overexpression. As shown in Fig. 2A, the mRNA expression levels of PTEN, Tet1, APOBEC3A, APOBEC3C and APOBEC3F 
were significantly decreased in the CagA overexpression group compared with in the negative control group $(\mathrm{P}<0.01)$. However, there were no significant differences regarding the expression of Ras, c-myc, c-met, ESR2, Tet2, Tet3, ACRC, AICDA, ALKBH1, ALKBH2, APEX1, APOBEC2, FTO or TDG in the cells (Fig. 2B).

Decreased expression of PTEN in HGC-27 cells with Tet1 interference is associated with its increased methylation. RT-qPCR and western blotting were used to detect the mRNA and protein expression levels of PTEN, respectively. Compared with in the negative control group, the mRNA and protein expression levels of PTEN were markedly decreased in HGC-27 cells with Tet1 interference $(\mathrm{P}<0.001$; Fig. 3A and B). The decreased expression of PTEN in the Tet1 interference group was associated with its increased methylation (Fig. 3C).

Decreased expression of PTEN in HGC-27 cells with CagA overexpression is reversed by Tetl overexpression. Compared with in the negative control group, the mRNA and protein expression levels of PTEN were markedly decreased in HGC-27 cells with CagA overexpression $(\mathrm{P}<0.001$; Fig. 4A and $\mathrm{B})$, which was significantly reversed by Tetl overexpression $(\mathrm{P}<0.01$; Fig. $4 \mathrm{~A}$ and $\mathrm{B})$. In addition, the decreased expression of PTEN in the CagA overexpression group was associated with its increased methylation in the cells, whereas Tet1 overexpression attenuated this methylation (Fig. 4C).

Expression of PTEN and Tet1 is markedly decreased in $\mathrm{CagA}^{+}$ gastric cancer tissues. According to the expression of $\mathrm{CagA}$, gastric cancer tissues were grouped into a $\mathrm{CagA}^{-}$or a $\mathrm{CagA}^{+}$ group (Fig. 5A). Immunohistochemistry was used to detect the expression of CagA. If CagA was expressed in the gastric cancer tissues, it was designated the $\mathrm{CagA}^{+}$group. If $\mathrm{Cag} \mathrm{A}$ was not expressed in the gastric cancer tissues, it was designated the CagA- group (data not shown). The results indicated that the expression levels of PTEN and Tet1 were significantly decreased in the $\mathrm{CagA}^{+}$gastric cancer tissues compared with in $\mathrm{CagA}^{-}$gastric cancer tissues (Fig. 5A and $\mathrm{B}$; $\mathrm{P}<0.05$ ). In addition, the decreased expression of PTEN in $\mathrm{CagA}^{+}$gastric cancer tissues was associated with its increased methylation (Fig. 5C).

\section{Discussion}

The present study demonstrated that CagA may significantly decrease the expression of PTEN, Tet1, APOBEC3A, APOBEC3C and APOBEC3F in human gastric cancer. The results suggested that $\mathrm{Cag} A$ decreased PTEN expression by increasing methylation levels, and decreased expression of PTEN was significantly attenuated by Tet1.

The risk of gastric cancer differs among various strains of H. pylori. Strains with virulence factors, including CagA and vacuolating cytotoxin A, are more likely to be associated with precancerous lesions and gastric cancer (15-17). A specific motif (AATAAGATA) has been revealed to be linked to the capacity of $H$. pylori to express CagA (18). Highly virulent strains of $H$. pylori produce CagA-modulated c-met receptor signal transduction pathways in vitro, which may influence the initiation and progression of gastric cancer (19).
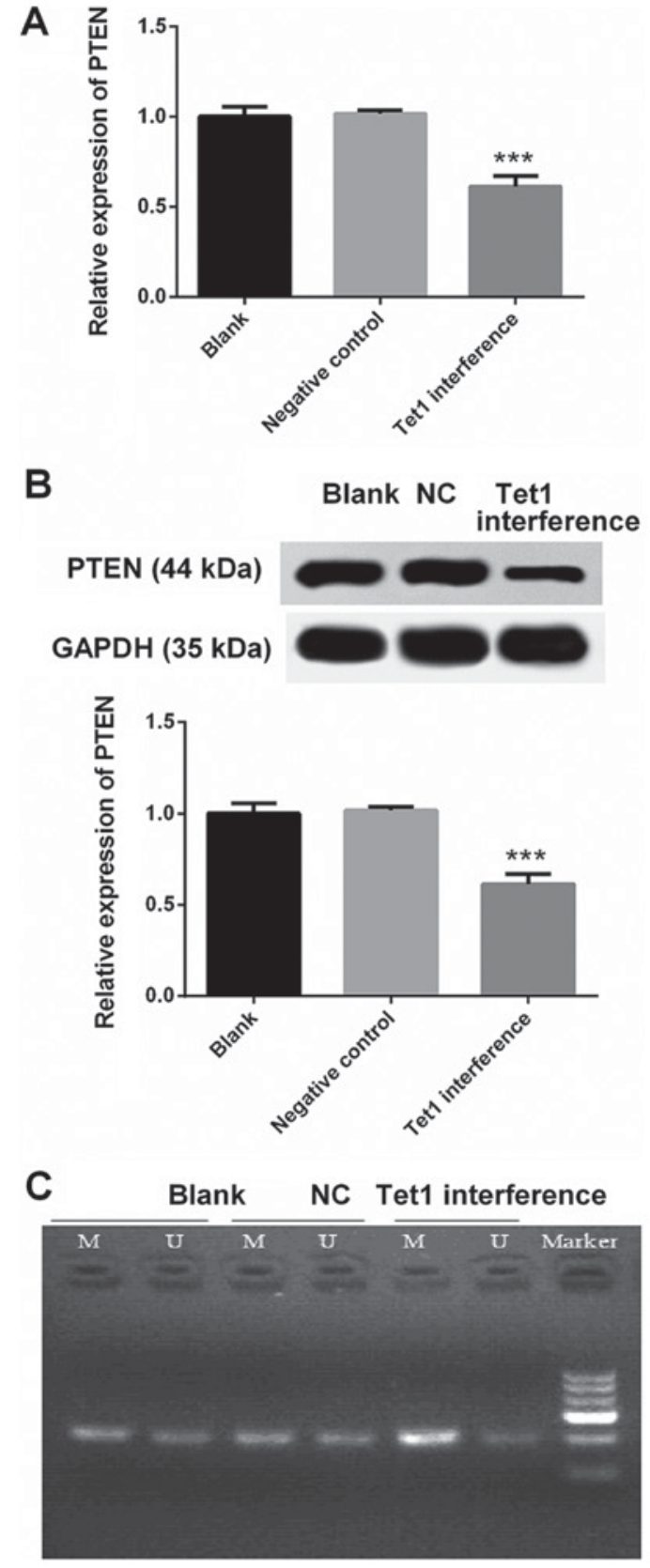

Figure 3. Decreased expression of PTEN in the Tet1 interference group is associated with its increased methylation. (A and B) HGC-27 cells were infected with viruses carrying a Tet1 interference vector. mRNA and protein expression levels of PTEN were detected in the cells using reverse transcription-quantitative PCR or western blotting, respectively. (C) The methylation status of PTEN was detected using methylation-specific PCR. ${ }^{* * * *} \mathrm{P}<0.001$ vs. the NC group ( $n=3)$. M, methylated; NC, negative control; PCR, polymerase chain reaction; PTEN, phosphatase and tensin homolog; Tet1, tet methylcytosine dioxygenase 1 ; U, unmethylated.

Gastric carcinogenesis has been proposed to involve an initial stage of dedifferentiation, including gastric atrophy, followed by abnormal redifferentiation, including intestinal metaplasia. This process is mediated by the effects of $H$.pylori infection, particularly $\mathrm{CagA}^{+}$strains, on $\beta$-catenin $(20,21)$. Apical junctions are disrupted by CagA expression, and mechanisms that maintain the differentiation of normal epithelium, including cell adhesion, cell polarity and inhibition of migration, are also disturbed by CagA expression in vitro (22). In addition, proteolytic cleavage of E-cadherin is induced, and 
A

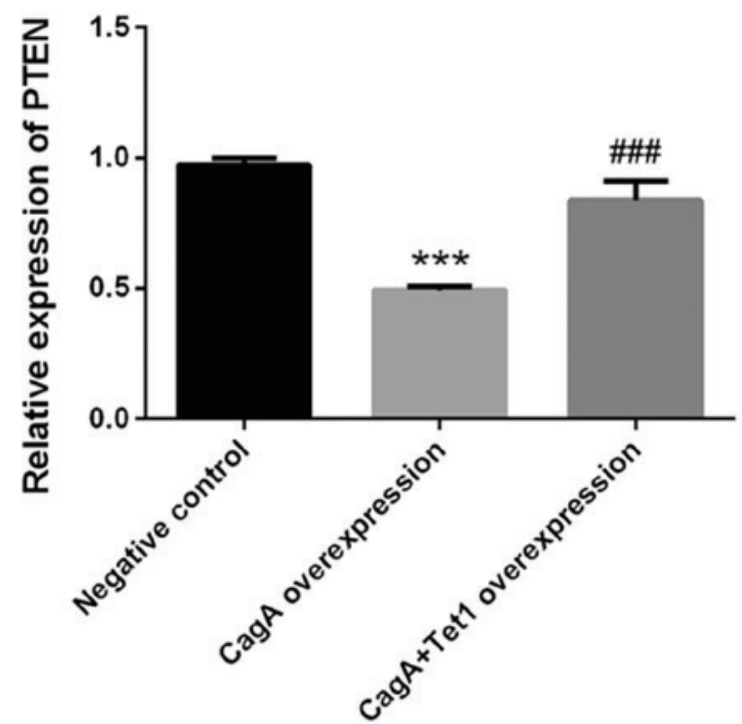

B

\section{PTEN (44 kDa) GAPDH (35 kDa)}

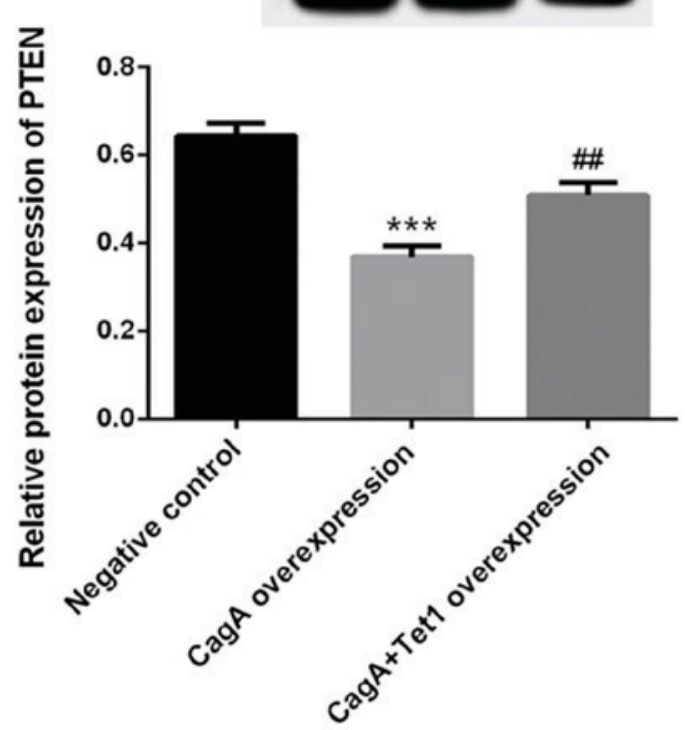

C

NC CagA CagA+Tet1

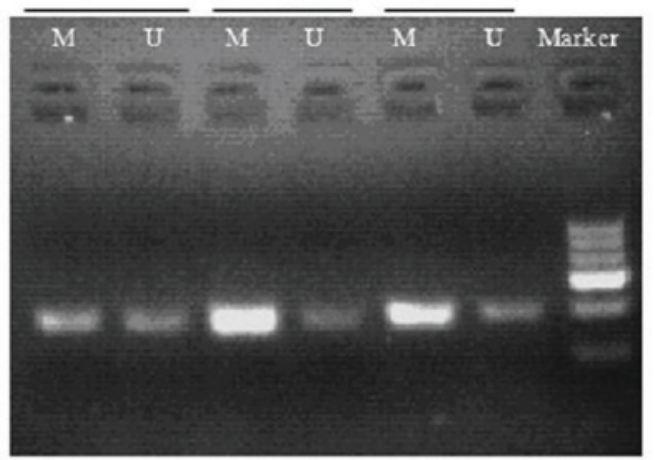

Figure 4. Decreased expression of PTEN in HGC-27 cells with CagA overexpression is reversed by Tet1 overexpression. HGC-27 cells were infected with viruses carrying CagA and/or a Tet1 overexpression vector. (A) mRNA expression levels of PTEN in the cells was detected by reverse transcriptionquantitative PCR. (B) Western blot analysis was used to measure the protein expression levels of PTEN in the cells. (C) Methylation status of PTEN was detected by methylation-specific PCR in HGC-27 cells. ${ }^{* * *} \mathrm{P}<0.001$ vs. the negative control group; ${ }^{\# \#} \mathrm{P}<0.01$, ${ }^{\# \# \#} \mathrm{P}<0.001$ vs. the CagA overexpression group $(n=3)$. M, methylated; PCR, polymerase chain reaction; PTEN, phosphatase and tensin homolog; Tet1, tet methylcytosine dioxygenase 1; U, unmethylated.

E-cadherin-dependent cell-to-cell contact is disrupted by CagA (23). Formation of the E-cadherin/ $\beta$-catenin complex is also impaired by $\mathrm{CagA}$, thus leading to the accumulation of $\beta$-catenin in the cytoplasm and nucleus, and constitutive transcription of intestinal differentiation markers. Nuclear localization of $\beta$-catenin has also been reported to be present in a carcinogenic human gastric cell line, but not in a non-carcinogenic clinical isolate $(6,24)$.

In the present study, a highly metastatic gastric cancer cell line, HGC-27, which was initially isolated from an Asian patient with gastric cancer, was used. This cell line is derived from a metastatic lymph node and may provide a novel perspective for the role of CagA in gastric cancer metastasis. The results of the present study demonstrated that CagA significantly decreased the expression levels of PTEN and Tet1 in this human gastric cancer cell line. In addition, it was revealed that CagA decreased PTEN expression by increasing its methylation level, which was significantly attenuated by Tet1. PTEN serves an important role in apoptosis, cell cycle progression and cell proliferation, and functions as a tumor-suppressor gene. It has been reported that Tet1 exerts its tumor-suppressor function in gastric cancer possibly via DNA demethylation (12). Tet1 has also been reported to inhibit the growth and metastasis of gastric cancer by demethylation and re-expression of PTEN. Tet1 suppresses the cell growth, migration and invasion of gastric cancer via demethylation of $\mathrm{CpG}$ islands in the PTEN promoter region, by increasing 5-hydroxymethylcytosine. The re-expressed PTEN has been revealed to downregulate the activity of AKT and focal adhesion kinase (25). This may explain how Tet1 attenuated CagA-induced methylation and PTEN downregulation in gastric cancer in the present study. The present findings have provided novel mechanisms for CagA-induced gastric carcinogenesis. 
A

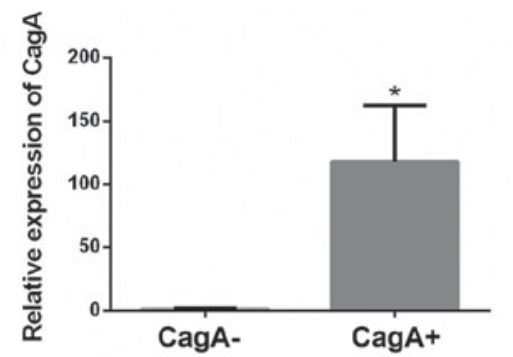

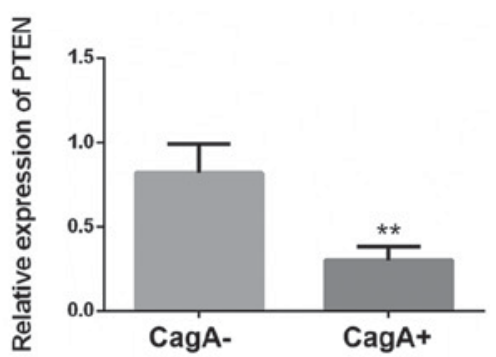

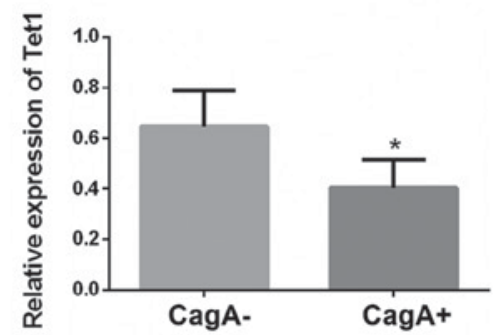

B

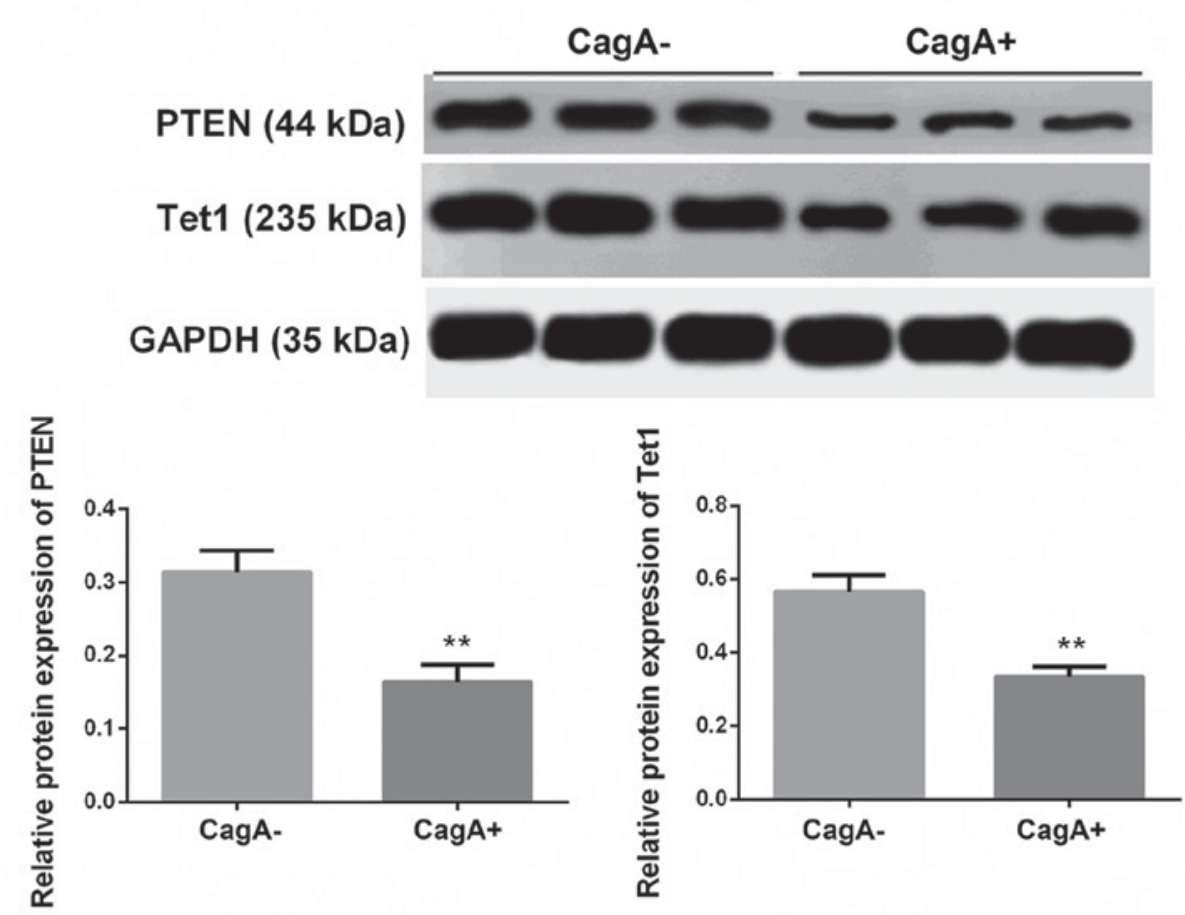

C

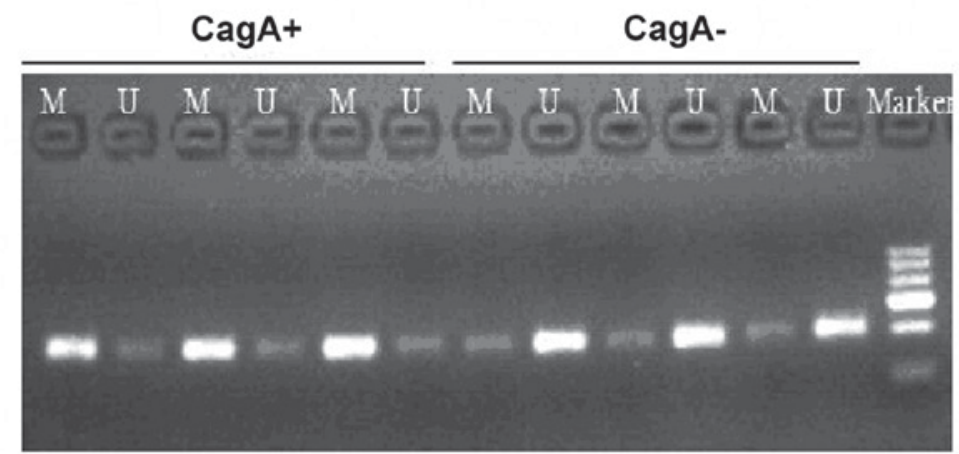

Figure 5. mRNA expression levels of PTEN and Tet1 were markedly decreased in Cag $\mathrm{A}^{+}$gastric cancer. (A) Gene expression levels of CagA, PTEN and Tet1 in $\mathrm{CagA}^{-}$and $\mathrm{CagA}^{+}$gastric tissues were detected with reverse transcription-quantitative PCR. (B) Western blotting was applied to measure the protein expression levels of PTEN and Tet1 in $\mathrm{CagA}^{-}$and $\mathrm{CagA}^{+}$gastric tissues. (C) Methylation status of PTEN in $\mathrm{CagA}^{-}$and $\mathrm{CagA}^{+}$gastric tissues was detected by methylation-specific PCR. ${ }^{*} \mathrm{P}<0.05$ and ${ }^{* *} \mathrm{P}<0.01$ vs. the CagA ${ }^{*}$ group $(\mathrm{n}=3)$. M, methylated; PCR, polymerase chain reaction; PTEN, phosphatase and tensin homolog; Tet1, tet methylcytosine dioxygenase 1; U, unmethylated.

The present study demonstrated that $\mathrm{Cag}$ A significantly decreased the expression levels of APOBEC 3A, APOBEC3C and $\mathrm{APOBEC} 3 \mathrm{~F}$ in human gastric cancer. APOBEC $3 \mathrm{~A}$ has been reported to possess anticancer and antiviral effects via inhibiting the expression of human papillomavirus E6 and E7 in cervical cancer (26). The deletion of APOBEC3A is also associated with a young age at diagnosis among patients with lung and prostate cancer (27). APOBEC $3 \mathrm{C}$ has been reported to be a potent inhibitor of replication of simian immunodeficiency virus (28), and APOBEC3F restricts the proliferation of human immunodeficiency virus (29). However, evidence regarding the roles of APOBEC3A, APOBEC $3 \mathrm{C}$ and $\mathrm{APOBEC} 3 \mathrm{~F}$ in human gastric cancer is scarce. To the best of our knowledge, the present study is the first to reveal that $\mathrm{CagA}$ decreased the expression levels of APOBEC 3A, APOBEC 3C and APOBEC3F in human gastric 
cancer. Although more research is required to elucidate the roles of APOBEC $3 \mathrm{~A}, \mathrm{APOBEC} 3 \mathrm{C}$ and $\mathrm{APOBEC} 3 \mathrm{~F}$ in gastric cancer, the present findings provide another explanation for CagA-induced gastric carcinogenesis. However, the results derived from studies of one cell line are not directly transferable to patients.

In conclusion, the present study demonstrated that CagA significantly decreased the expression of PTEN, Tet1, APOBEC 3A, APOBEC 3C and APOBEC3F in human gastric cancer cells. In addition, CagA decreased PTEN expression by increasing its methylation levels, which was significantly reversed by Tet1 upregulation. Further investigations are required to provide information on additional molecular mechanisms; however, the present study may precede future therapeutic approaches targeting human gastric cancer.

\section{Acknowledgements}

Not applicable.

\section{Funding}

The present study was supported by grants from the National Natural Science Foundation of China (grant no. 81602160), the Shandong Provincial Natural Science Foundation (grant no. ZR2016HL30), the Scientific Research Foundation for $\mathrm{PhD}$, Affiliated Hospital of Jining Medical University (grant no. 2016-BS-006), and the Project of Health and Family Planning Commission of Shandong province (grant no. 2017WS513).

\section{Availability of data and materials}

All data sets used in this study are available from the corresponding author on reasonable request.

\section{Authors' contributions}

DG and SL designed the study. BZ, XZ, MJ and LH performed the experiments and $\mathrm{MZ}, \mathrm{WQ}, \mathrm{SW}$ and $\mathrm{BL}$ analyzed the data. BZ, XZ and $\mathrm{MJ}$ were the major contributors in developing the first draft of this manuscript. DG and SL reviewed and approved the final draft of the manuscript prior to submission.

\section{Ethics approval and consent to participate}

All patients provided written informed consent and the study was approved by the Institutional Review Board/Ethics Committee of the Affiliated Hospital of Jining Medical University.

\section{Patient consent for publication}

All patients provided written informed consent for the publication of all associated data in this study.

\section{Competing interests}

The authors declare that they have no competing interests.

\section{References}

1. Zhu AL and Sonnenberg A: Is gastric cancer again rising? J Clin Gastroenterol 46: 804-806, 2012.

2. Correa P: Gastric cancer: Two epidemics? Dig Dis Sci 56: 1585-1586; author reply 1586, 2011.

3. Jeyamani L, Jayarajan J, Leelakrishnan V and Swaminathan M: CagA and VacA genes of Helicobacter pylori and their clinical relevance. Indian J Pathol Microbiol 61: 66-69, 2018.

4. Weel JF, van der Hulst RW, Gerrits Y, Roorda P, Feller M, Dankert J, Tytgat GN and van der Ende A: The interrelationship between cytotoxin-associated gene A, vacuolating cytotoxin, and Helicobacter pylori-related diseases. J Infect Dis 173: 1171-1175, 1996.

5. Huang JQ, Zheng GF, Sumanac K, Irvine EJ and Hunt RH: Meta-analysis of the relationship between cag A seropositivity and gastric cancer. Gastroenterology 125: 1636-1644, 2003.

6. Franco AT, Israel DA, Washington MK, Krishna U, Fox JG, Rogers AB, Neish AS, Collier-Hyams L, Perez-Perez GI, Hatakeyama M, et al: Activation of beta-catenin by carcinogenic Helicobacter pylori. Proc Natl Acad Sci USA 102: 10646-10651, 2005.

7. Basso D, Zambon CF, Letley DP, Stranges A, Marchet A, Rhead JL, Schiavon S, Guariso G, Ceroti M, Nitti D, et al: Clinical relevance of Helicobacter pylori cagA and vacA gene polymorphisms. Gastroenterology 135: 91-99, 2008.

8. Keniry M and Parsons R: The role of PTEN signaling perturbations in cancer and in targeted therapy. Oncogene 27: 5477-5485, 2008.

9. Krymskaya VP and Goncharova EA: PI3K/mTORC1 activation in hamartoma syndromes: Therapeutic prospects. Cell Cycle 8: 403-413, 2009.

10. Sansal I and Sellers WR: The biology and clinical relevance of the PTEN tumor suppressor pathway. J Clin Oncol 22: 2954-2963, 2004.

11. Stambolic V, Suzuki A, de la Pompa JL, Brothers GM, Mirtsos C, Sasaki T, Ruland J, Penninger JM, Siderovski DP and Mak TW: Negative regulation of PKB/Akt-dependent cell survival by the tumor suppressor PTEN. Cell 95: 29-39, 1998.

12. Fu HL, Ma Y, Lu LG, Hou P, Li BJ, Jin WL and Cui DX: TET1 exerts its tumor suppressor function by interacting with p53-EZH2 pathway in gastric cancer. J Biomed Nanotechnol 10: 1217-1230, 2014.

13. Pastor WA, Aravind L and Rao A: TETonic shift: Biological roles of TET proteins in DNA demethylation and transcription. Nat Rev Mol Cell Biol 14: 341-356, 2013.

14. Livak KJ and Schmittgen TD: Analysis of relative gene expression data using real-time quantitative PCR and the 2(-Delta Delta C(T)) method. Methods 25: 402-408, 2001.

15. Blaser MJ, Perez-Perez GI, Kleanthous H, Cover TL, Peek RM, Chyou PH, Stemmermann GN and Nomura A: Infection with Helicobacter pylori strains possessing cagA is associated with an increased risk of developing adenocarcinoma of the stomach. Cancer Res 55: 2111-2115, 1995.

16. Miehlke S, Kirsch C, Agha-Amiri K, Günther T, Lehn N, Malfertheiner P, Stolte M, Ehninger G and Bayerdörffer E: The Helicobacter pylori vacA $\mathrm{s} 1, \mathrm{~m} 1$ genotype and cagA is associated with gastric carcinoma in Germany. Int J Cancer 87: 322-327, 2000.

17. Plummer M, van Doorn LJ, Franceschi S, Kleter B, Canzian F, Vivas J, Lopez G, Colin D, Muñoz N and Kato I: Helicobacter pylori cytotoxin-associated genotype and gastric precancerous lesions. J Natl Cancer Inst 99: 1328-1334, 2007.

18. Loh JT, Shaffer CL, Piazuelo MB, Bravo LE, McClain MS, Correa P and Cover TL: Analysis of cagA in Helicobacter pylori strains from Colombian populations with contrasting gastric cancer risk reveals a biomarker for disease severity. Cancer Epidemiol Biomarkers Prev 20: 2237-2249, 2011.

19. Churin Y, Al-Ghoul L, Kepp O, Meyer TF, Birchmeier W and Naumann M: Helicobacter pylori CagA protein targets the c-Met receptor and enhances the motogenic response. J Cell Biol 161: 249-255, 2003

20. Faller $\mathrm{G}$ and Kirchner T: Immunological and morphogenic basis of gastric mucosa atrophy and metaplasia. Virchows Arch 446: $1-9,2005$.

21. Hlubek F, Spaderna S, Schmalhofer O, Jung A, Kirchner T and Brabletz T: Wnt/FZD signaling and colorectal cancer morphogenesis. Front Biosci 12: 458-470, 2007. 
22. Bagnoli F, Buti L, Tompkins L, Covacci A and Amieva MR: Helicobacter pylori CagA induces a transition from polarized to invasive phenotypes in MDCK cells. Proc Natl Acad Sci USA 102: 16339-16344, 2005.

23. Weydig C, Starzinski-Powitz A, Carra G, Löwer J and Wessler S: CagA-independent disruption of adherence junction complexes involves E-cadherin shedding and implies multiple steps in Helicobacter pylori pathogenicity. Exp Cell Res 313: 3459-3471, 2007.

24. Murata-Kamiya N, Kurashima Y, Teishikata Y, Yamahashi Y, Saito Y, Higashi H, Aburatani H, Akiyama T, Peek RM Jr, Azuma T and Hatakeyama M: Helicobacter pylori CagA interacts with E-cadherin and deregulates the beta-catenin signal that promotes intestinal transdifferentiation in gastric epithelial cells. Oncogene 26: 4617-4626, 2007.

25. Pei YF, Tao R, Li JF, Su LP, Yu BQ, Wu XY, Yan M, Gu QL, Zhu ZG and Liu BY: TET1 inhibits gastric cancer growth and metastasis by PTEN demethylation and re-expression. Oncotarget 7: 31322-31335, 2016.
26. Chen S, Li X, Qin J, Chen Y, Liu L, Zhang D, Wang M, Wang M and Zhang D: APOBEC3A possesses anticancer and antiviral effects by differential inhibition of HPV E6 and E7 expression on cervical cancer. Int J Clin Exp Med 8: 10548-10557, 2015.

27. Gansmo LB, Romundstad P, Hveem K, Vatten L, Nik-Zainal S, Lønning PE and Knappskog S: APOBEC3A/B deletion polymorphism and cancer risk. Carcinogenesis 39: 118-124, 2017.

28. Yu Q, Chen D, König R, Mariani R, Unutmaz D and Landau NR: APOBEC3B and APOBEC3C are potent inhibitors of simian immunodeficiency virus replication. J Biol Chem 279: 53379-53386, 2004.

29. Ara A, Love RP, Follack TB, Ahmed KA, Adolph MB and Chelico L: Mechanism of enhanced HIV restriction by virion coencapsidated cytidine deaminases APOBEC3F and APOBEC3G. J Virol 91: e02230-16, 2016.

c) (i) $(9$ This work is licensed under a Creative Commons cc) Attribution-NonCommercial-NoDerivatives 4.0 International (CC BY-NC-ND 4.0) License. 